\title{
7e Symposium universitaire canadien en optométrie
}

\section{PAR ETTY BITTON, OD, MSC, FAAO}

$\mathrm{D}$ es membres du corps professoral et des étudiants des deux écoles d'optométrie du Canada se réunissent de temps à autre pour échanger, réseauter et se rendre visite afin de demeurer en contact. L'École d'optométrie de l'Université de Montréal (UM) a accueilli cette année le $7^{\mathrm{e}}$ Symposium universitaire canadien en optométrie qui a eu lieu en décembre 2010 à Montréal. Le premier de ces échanges remonte à 1988 à Montréal. Le Dr Jacques Gresset, directeur de l'UM, et le Dr Thomas Freddo, directeur de l'École d'optométrie de l'Université de Waterloo (UW) à ce moment, ont tous deux prononcé un discours d'ouverture. Plus de 200 étudiants en optométrie, étudiants du deuxième cycle, cliniciens et membres du corps professoral des deux écoles ont pu échanger et participer à cet événement spécial.

\section{Le lien entre Montréal et Waterloo}

Les deux écoles sont intimement liées depuis des années, non seulement parce qu'elles offrent les deux seuls programmes d'optométrie au Canada, mais aussi parce que beaucoup des membres du corps professoral ont des liens avec les deux établissements. Jusqu'à maintenant, neuf personnes ont eu des liens avec les deux établissement.
Le Dr Jacob Sivak était diplômé de l'UM lorsqu'il a terminé sa formation en optométrie. Il s'est joint par la suite à Waterloo comme membre du corps professoral et il est devenu directeur de l'école (1984-1990 et 1993-1996) et ensuite, doyen de la Faculté des sciences (1999 à 2002). En novembre 2000, l'UM l'a honoré en lui décernant un diplôme honorifique pour ses nombreuses contributions à la science de la vision. Le Dr Sivak a toujours un laboratoire de recherche très actif et demeure professeur émérite à Waterloo.

Le Dr John V. Lovasik, diplômé en optométrie de Waterloo, y est demeuré pendant plusieurs années après avoir terminé des études supérieures et s'être joint au corps professoral. Le Dr Lovasik a ensuite déménagé à Montréal pour devenir directeur de l'École de l'UM (1989-1995). Il y demeure membre du corps professoral comme professeur titulaire.

La Dre Angela Kothe a reçu son diplôme en optométrie de Waterloo. Elle a poursuivi ses études de deuxième cycle sous la direction du Dr Lovasik à l'UW. Elle s'est jointe par la suite au corps professoral de l’UM de 1988 à 1995 et a été chargée de mettre à niveau la partie du programme d'études portant sur les maladies oculaires. La
Dre Kothe travaille actuellement dans l'industrie.

\section{La Dre Hélène Kergoat a ob-} tenu son diplôme en optométrie de l'UM. Après avoir pratiqué pendant plusieurs années, elle a décidé de poursuivre ses études au niveau supérieur et les a complétées à Waterloo sous la direction du Dr Lovasik. La Dre Kergoat est ensuite revenue à Montréal où elle s'est jointe au corps professoral et demeure active en recherche clinique.

Le Dr Pierre Simonet, diplômé de l'UM, a suivi une voie semblable et complété des études supérieures en optique à Waterloo, sous la direction de la Dre Melanie Campbell. Il est retourné à Montréal comme professeur et a dirigé l'école de 1996 à 2003. Le Dr Simonet est ensuite passé à l'administration de l'Université comme directeur de la planification (2003 à 2005) et il est devenu vice-recteur de l'Université de 2005 à 2010.

La Dre Etty Bitton, diplômée de Waterloo, est revenue pratiquer à Montréal. Après avoir obtenu un diplôme de deuxième cycle à l'UM sous la direction du Dr Lovasik, elle s'est jointe au corps professoral à temps plein. Elle est retournée pour un congé sabbatique à Waterloo où elle a collaboré avec 
le corps professoral de l'UW à différents projets portant sur le film lacrymal. La Dre Bitton demeure membre du corps professoral de l'UM comme professeure agrégée et directrice du Programme des stages externes.

Le Dr Daniel Boissy, diplômé de l'UW, est revenu à Montréal pour se joindre au cabinet de son père, situé en périphérie de la ville. Le Dr Boissy est aussi chargé de cours à l'UM, enseigne le laboratoire sur les lentilles de cornéennes et est aussi clinicien à la clinique générale.

\section{La Dre Sharon Wong a com-}

plété ses études supérieures sous la direction du Dr Jacob Sivak à l'UW. Inspirée par la possibilité de jumeler la recherche fondamentale aux applications cliniques, elle s'est inscrite en optométrie à l'UM. Peu après avoir obtenu son diplôme, la Dre Wong a déménagée en Colombie-Britannique où elle pratique actuellement.

\section{Le Dr Thomas Freddo,}

Directeur (2005-2011) de l'École d'optométrie de l'UW, a été honoré, au cours de la collation des grades de 2010, par l'UM qui lui a décerné un diplôme honorifique pour ses nombreuses contributions aux mécanismes biochimiques, histologiques et vasculaires de l'écoulement de l'humeur aqueuse.
Il est facile de comprendre comment ces personnes ont eu des liens étroits avec les deux établissements et qu'elles continueront de promouvoir les mérites des deux écoles d'optométrie du Canada. Ces réunions continuent de favoriser et de resserrer les liens étroits qui existent entre les deux établissements, aujourd'hui et demain. Qui sera la prochaine personne à se déplacer entre les deux établissements et à bénéficier de ce que les deux ont à offrir ?

\section{Points saillants du 7e Symposium}

Les conférenciers d'honneur des deux établissements ont décrit une partie des recherches fondamentales et cliniques qu'ils ont effectuées dans leur domaine respectif. Voici un résumé des conférences qu'ils ont prononcées.

Aperçus de la pénombre : I'anatomie de la cécité

\section{Maurice Ptito, PhD, FAAO} Professeur, UM

La privation de la vue depuis la naissance entraîne une restructuration anatomique du cerveau provoquée par l'apprentissage. Nous avons utilisé des techniques comportementales pour démontrer que des personnes, vivant avec une cécité congénitale, peuvent traiter des stimulis visuels au moyen du système somatosensoriel. À cette fin, elles mettent à contribution leur cortex visuel et ses flots efférents (ventral et dorsal). On a utilisé des techniques d'imagerie du cerveau pour démontrer que le cerveau de la personne aveugle subit des changements anatomiques et métaboliques massifs qui aboutissent à la réorganisation de la somatotopie à la fois de la langue et des doigts. Dans sa conférence, le Dr Ptito a présenté un aperçu des voies anatomiques utilisées pour atteindre le cortex visuel en l'absence totale d'entrées visuelles.

\section{CSEye Waterloo :}

Les tribulations d'un optométriste judiciaire Graham Strong, OD, MSC Professeur, UW

Les détectives de l'escouade des homicides découvrent une paire de lunettes tordues sous un corps sur la scène d'un crime. Ils ont hâte de savoir si les lunettes appartiennent à la victime, à son assassin, à un témoin possible ou à quelqu'un qui n'a absolument rien à voir avec l'homicide, mais qui a perdu ses lunettes par inadvertance à un endroit qui est devenu la scène d'un crime. Graham Strong, professeur d'optométrie à l'UW, est consulté périodiquement pour aider à résoudre de tels cas en analysant les preuves ophtalmiques qui font leur apparition au cours de l'enquête et des poursuites subséquentes. Le Dr Strong a décrit aux participants la science curieuse que constitue « l'optométrie judiciaire » en faisant allusion précisément à plusieurs homicides difficiles qu'il a aidé à résoudre au cours des 20 dernières années. 


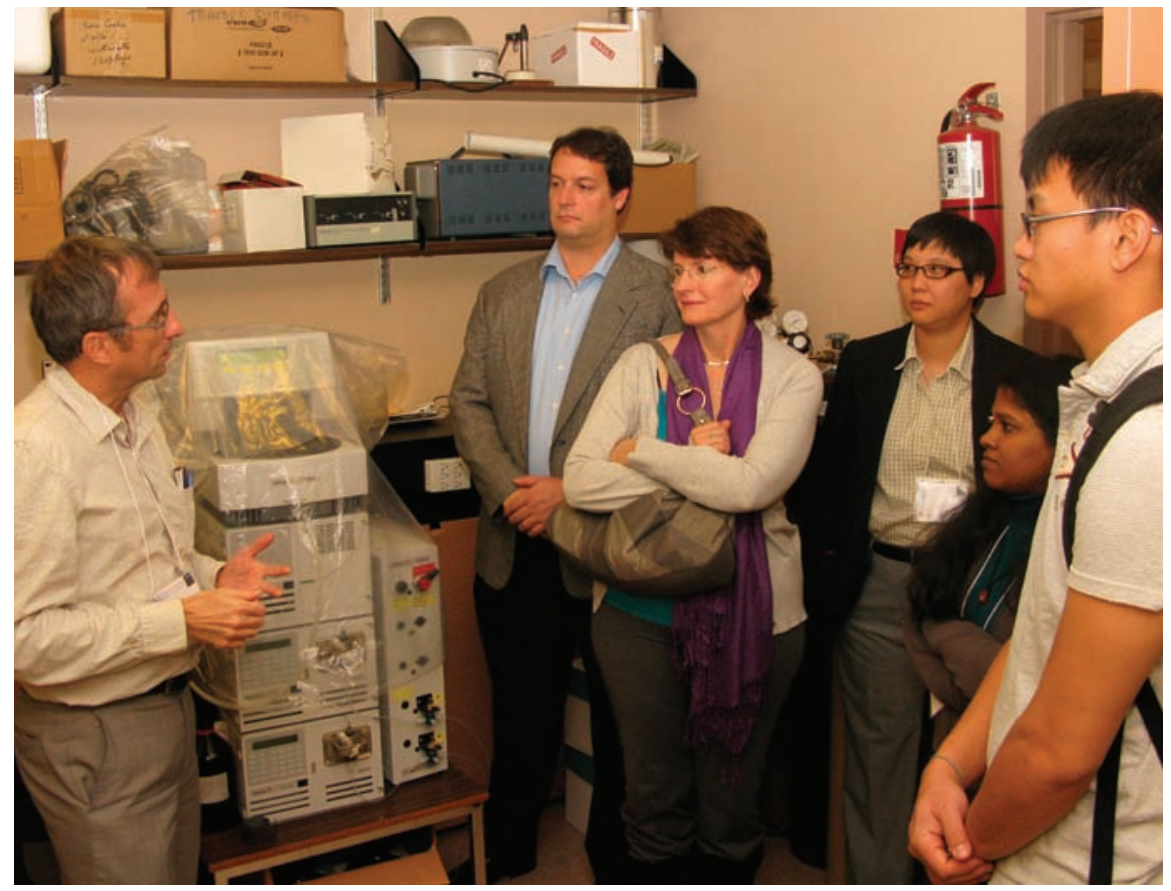

Le Dr Claude Giasson reçoit les participants dans son laboratoire de physiologie cornéenne.

\section{Le couplage neurovasculaire de rétine humaine John Lovasik, OD, PhD, FAAO Professeur, UM}

Le « couplage neurovasculaire » (CNV) s'entend du phénomène physiologique par lequel l'activation des neurones accroit l'apport sanguin dans le tissu cible afin d'appuyer l'élévation du métabolisme.

Au niveau de la rétine, des mécanismes d'autorégulation du métabolisme et de la pression régularisent la circulation sanguine par la vasoconstriction ou la vasodilatation. Il est maintenant possible de mesurer avec précision, en temps réel et in vivo, les changements de la régulation en déterminant le calibre des vaisseaux au moyen d'un analyseur des vaisseaux de la rétine (par Imedos).
Le Dr Lovasik a décrit ses études sur la régulation de la circulation sanguine dans les systèmes vasculaires choroïdien et rétinien, ainsi que de CNV dans les capillaires qui alimentent la tête du nerf optique. Le Dr Lovasik a conclu son exposé en décrivant les répercussions cliniques de ces études et leur pertinence pour l'optométrie.

\section{Découverte des causes de la myopie et sa prévention: nature vs milieu Jacob Sivak, LScO, PhD, OD, FAAO Professeur, UW}

La recherche sur les causes de la myopie et sa prévention a une histoire longue et controversée. C'est un problème qui attire énormément d'attention depuis presque 200 ans à cause de sante de la myopie et de sa nature l'incidence importante et crois- évolutive chez les enfants. Dès le milieu du XIIX siècle, Donders et Helmholtz posaient en hypothèse l'existence possible d'un lien entre le travail rapproché et la myopie. Des divergences de vues quant à l'importance relative de la génétique par rapport à l'environnement ont toutefois caractérisé une bonne partie de la discussion sur la myopie pendant la majeure partie du $\mathrm{XX}^{\mathrm{e}}$ siècle. La mise au point de plusieurs modèles animaux d'apparition des erreurs de réfraction a toutefois mis en évidence le rôle de l'environnement depuis 30 ans. En dépit d'efforts répétés toujours plus minutieux et complexes, y compris une récente évaluation multicentrique de l'usage possible de lentilles d'appoint progressives chez les enfants, les chercheurs n'ont néanmoins pas réussi à établir un lien clair et sans équivoque entre l'accommodation excessive et l'apparition de la myopie. Dans son exposé, le Dr Sivak a discuté de la recherche historique et en cours sur l'apparition de la myopie chez les enfants qui a porté avant tout sur le rôle des activités de plein air, la myopie des parents, l'ésophorie, le retard de l'accommodation, ainsi que sur l'usage possible de médicaments cycloplégiques. 


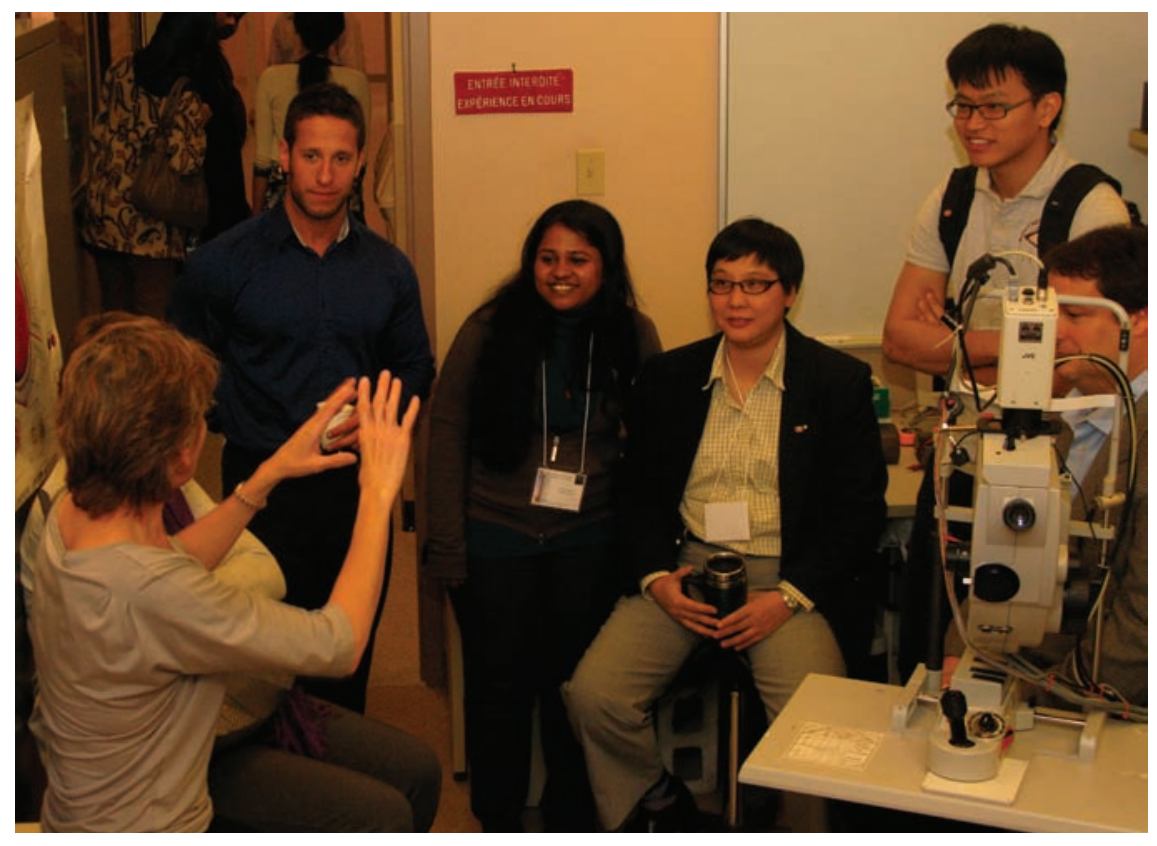

Le Dre Kergoat, professeure de I'UdeM, présente sa recherche dans le domaine de la gériatrie aux participants.

Est-ce qu'il y aura des lentilles cornéennes en 2020 ?

Lyndon Jones, PhD, FCOptom, FAAO (Dip CL), Professeur, UW

En dépit des progrès importants réalisés depuis 20 ans sur le plan des matériaux qui servent à fabriquer les lentilles cornéennes et sur celui des systèmes d'entretien, beaucoup de patients ont toujours de la difficulté à trouver un confort soutenu avec leurs lentilles. Les professionnels continueront-ils d'ajuster des lentilles au cours des 10 prochaines années, pendant que la technologie des lunettes et la chirurgie réfractive continuent de progresser? Le cabinet d'optométrie de 2020 se donnera$\mathrm{t}$-il la peine de recommander des lentilles aux patients? Le Dr Jones a passé en revue les recherches les plus récentes sur les lentilles cornéennes et a ouvert une fenêtre sur l'avenir pour laisser entrevoir ce que les lentilles cornéennes de 2020 auront à offrir aux patients.

Les obstacles à la réadaptation de la vision : I'histoire de Montréal Olga Overbury, PhD Professeure agrégée, UM

Beaucoup de personnes qui ont une déficience de la vision ont droit à des services de réadaptation et en ont besoin mais ne les reçoivent jamais. L'étude de Montréal sur les obstacles constitue une recherche multicentrique visant à créer une base de données qui aidera à déterminer les facteurs qui pourraient empêcher les personnes qui ont une basse vision d'obtenir les appareils fonctionnels et les interventions dont elles ont besoin pour optimiser leur fonction visuelle. Plus de 700 personnes participent à l'étude jusqu'à maintenant. On discutera de leurs caractéristiques démographiques et psychologiques en fonction de leur utilisation des services d'aide à la faible vision. Ces caractéristiques ont fait l'objet de discussions dans le contexte des tendances des références des professionnels de la vue et de celles du processus décisionnel chez les personnes ayant des troubles de la vision.

\section{Adaptation et satisfaction des} lentilles progressives

\section{Natalie Hutchings, PhD, MCOptom, Professeure adjointe, UW}

Les lentilles d'addition progressives (PAL) sont un moyen de correction populaire chez les personnes qui ont de la presbytie, mais nous savons peu de choses sur l'expérience du rendement d'une lentille vécue par les porteurs et sur le lien entre l'expérience et la conception des lentilles. La technologie de conception et de fabrication de ces lentilles a progressé très rapidement et c'est pourquoi il existe maintenant une multitude de lentilles "personnalisées » au sujet desquelles on affirme sélectionner le concept en fonction du comportement visuel de la personne qui les porte, de ses habitudes de vie ou du profil de ses aberrations oculaires. Les caractéristiques de la conception reposent toutefois avant tout sur des concepts théoriques et en apprenant à mieux connaittre le rendement subjectif de ces lentilles, nous pourrons déterminer s'il y a vraiment des 
avantages à les personnaliser ou à choisir un concept approprié pour une personne en particulier. La Dre Hutchings a présenté ses constatations tirées de tout un éventail d'expériences conçues pour comprendre le changement des mouvements de l'œil et de la tête durant l'adaptation chez les nouveaux porteurs de lentilles d'appoint progressives, ainsi que de l'expérience subjective correspondant à tout un éventail de principes différents de conception de lentilles. L'adaptation aux PAL a semblé prendre plus de quatre semaines chez les membres du groupe examinés $(\mathrm{n}=10)$. Au cours de l'adaptation, le groupe a effectué davantage de mouvements de la tête, ce qui était des plus évidents au cours de la lecture. Cette constatation n'était toutefois pas différente entre les deux types de lentilles étudiées, mais elle concorde avec les publications qui indiquent que l'on est plus susceptible de bouger la tête lorsque la trajectoire vers le stimulus suivant est connue, comme dans le cas de la lecture. Sur le plan de la satisfaction, le confort relié aux tâches a semblé s'améliorer en fonction de la personnalisation des lentilles. Des évaluations subjectives analysées au moyen d'un arbre de régression ont aussi permis d'établir une distinction entre les sujets "globalement satisfaits », « globalement insatisfaits » et « neutres » face au rendement perçu avec des lentilles personnalisées différentes. Même si les facteurs et leur évaluation relative différaient selon les principes de conception, le défi à venir consistera à établir un lien entre les caractéristiques de conception précises qui ont une incidence sur ces facteurs et leur évaluation.

Le câblage du système neurovisuel : Le rôle des cannabinoïdes au cours du développement

\section{Jean-François Bouchard, PhD Professeur agrégé, UM}

La vision est un de nos sens les plus importants. Les coûts socioéconomiques associés à l'absence ou à la perte des capacités visuelles sont astronomiques.

Les déficiences neurovisuelles ont plusieurs causes : elles peuvent être congénitales (hypoplasie ou atrophie du nerf optique), posttraumatiques, dégénératives (dégénérescence maculaire) ou découler d'autres pathologies (glaucome, diabète, etc). Ces pathologies sont actuellement incurables. L'identification des médiateurs qui interviennent dans le guidage et la synaptogénèse des axones visuels constitue donc un moyen précieux à utiliser pour mettre au point de nouveaux agents thérapeutiques afin de traiter ces maladies incu-rables. Dans le cerveau adulte, les endocannabinoïdes (eCB) exercent une fonction neuromodulatrice importante en agissant comme messagers rétrogrades pour régulariser la fonction de nombreux synapses. De plus en plus de preuves incriminent les eCB et leurs récepteurs dans plusieurs événements liés au développement, comme la prolifération et la migration des cellules, le guidage des axones et la synaptogénèse. Le Dr Bouchard décrit l'effet des eCB sur le guidage et la synaptogénèse des axones et des cellules ganglionnaires rétiniennes (CGR). L'étude des médiateurs mis en cause durant le développement neurovisuel nous permettra de déterminer de nouveaux objectifs pharmacologiques visant à régénérer et à reconstruire les voies visuelles.

\section{Reconnaissance spéciale}

Depuis plus d'une décennie, le Centre de recherche en lentille cornéennes (CCLR) contribue à l'avancement de la recherche et de l'éducation sur les lentilles cornéennes (LC) et il est maintenant reconnu sur la scène internationale pour ses nombreuses publications. L'UM a présenté une plaque pour rendre hommage au directeur du CCLR, le Dr Desmond Fonn, ainsi qu'au CCLR, pour leurs nombreu-ses contributions à la recherche et à l'éducation sur les LC, ainsi qu'à l'optométrie canadienne. Le Dr Freddo et le Dr Jones ont reçu la plaque au nom du Dr Fonn, qui ne pouvait être présent.

\section{Présentations scientifiques}

Les séances d'affiches ont constitué aussi un des points saillants du programme mettant en vedette à la fois la recherche fondamentale et la recherche clinique d'étudiants 


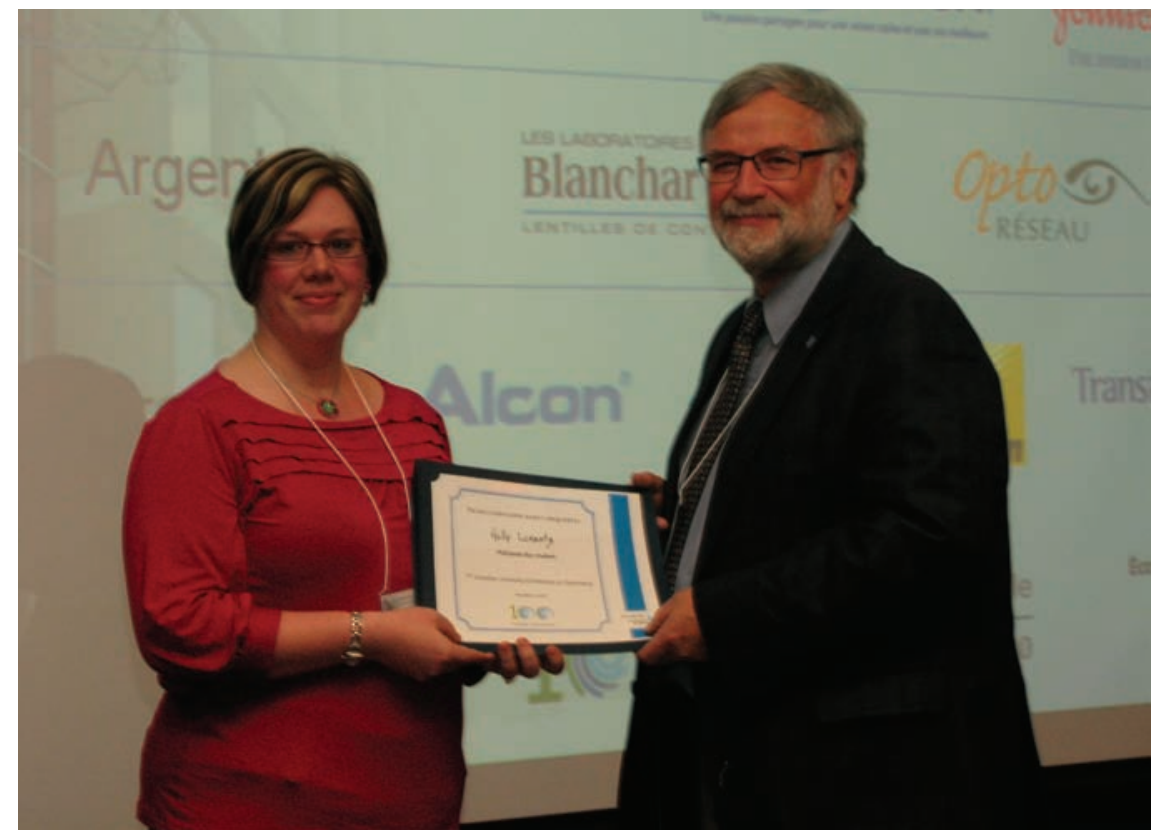

Le Dr. Gresset, Directeur de L'UdeM présente un certificat pour le meilleur affiche de la catégorie d'étude de $3^{e}$ cycle a Holly Lorentz de UW.

en optométrie, d'étudiants de cycle supérieurs et de membres du corps professoral des deux établissements. Le Tableau 1 résume les affiches présentées au cours du symposium.

Les juges, travaillant en équipes de deux, soit un de l'UM et un de l'UW, ont interrogé chaque présentateur au sujet de sa recherche respective et ont désigné deux gagnants à la fin de la journée. Le gagnant dans la catégorie meilleure affiche par un étudiant en optométrie ou étudiant au $2^{\mathrm{e}}$ cycle, a été Bruno Cécyre de l'UM pour la présentation intitulée « Les endocannabinoïdes modulent le guidage des axones et la sélection des cibles durant le développement du système de vision ", dont les auteurs sont B. Cécyre, G. Duff, A. Argaw, $\mathrm{N}$. Tea et Jean-François Bouchard.
La présentation intitulée «L'effet des éléments du film lacrymal sur l'absorption in vitro des lipides dans les lentilles cornéennes à base d'hydrogel et de silicone d'hydrogel », dont les auteurs, soit Holly Lorentz, Miriam Heynen et Lyndon Jones de l'UW, ont mérité le prix dans la catégorie étudiant au niveau du doctorat ou postdoctoral.

\section{Autres activités}

Des étudiants ambassadeurs en optométrie de l'UM étaient disponibles pour faire visiter l'école d'optométrie le deuxième jour du symposium. Les intéressés ont visité la clinique, la préclinique, la bibliothèque, le salon des étudiants et les laboratoires de recherche.

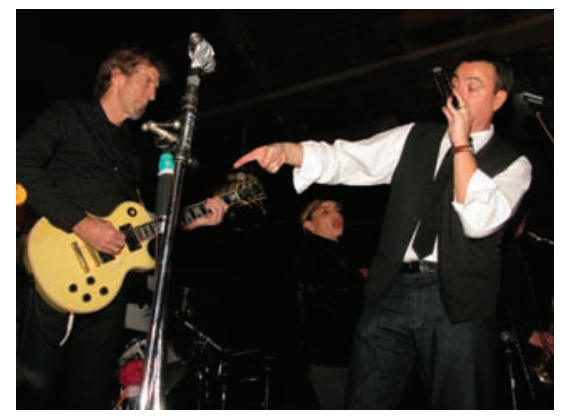

L'orchestre « Lost Faculties » anime une des soirées du symposium.

Les participants ont pu échanger tout au long de l'événement, au cours des conférences, des séances d'affiches et des repas. Une des soirées a mis en vedette le son des «Lost Faculties », orchestre constitué de plusieurs membres du corps professoral de l'UW. Les chanteurs du groupe étaient les conférenciers vedettes du symposium, ce qui montre les talents divers des membres du corps professoral! La socialisation que nous permettent ces événements « après les heures » est tout aussi importante pour réunir les gens et créer des souvenirs.

Bien entendu, rien de cela n'aurait été possible sans le travail de personnes dévouées qui ont participé à l'organisation du symposium. Nous remercions tous les participants et ceux qui ont contribué à la réussite de la réunion, ainsi que l'UM, d'avoir accueilli le $7^{\mathrm{e}}$ Symposium universitaire canadien en optométrie. Nous avons hâte à la prochaine réunion à Waterloo, dans quelques années. 